\title{
El conocimiento indígena en los niños: una experiencia de evocación y organización para el aula Indigenous Knowledge in Children:
} An Experience of Evocation and Organization
for the Classroom

\author{
María Luisa Matus Pineda \\ Escuela Superior Nido de Sócrates, El Espinal, Oaxaca, México \\ luisa_m21@hotmail.com
}

\section{RESUMEN}

El artículo da a conocer la experiencia en la aplicación de estrategias para la evocación del conocimiento indígena relacionado con la pesca, presente en niños de una escuela primaria bilingüe ikoot de San Mateo del Mar, en el estado de Oaxaca, México. Lo anterior se logró por medio de estrategias inductivas evocadoras de los conocimientos de los niños y de las niñas a través de la sintaxis cultural expresada en el Método Inductivo Intercultural de Jorge Gashé (2008). Algunos de los resultados son la aproximación a la explicitación y organización del conocimiento indígena, y el acercamiento al tema de la observación tácita como una forma de aprendizaje valiosa que es utilizada en contextos socioculturalmente diversos.

Palabras clave: explicitación, conocimiento indígena, estrategias evocadoras, educación

\begin{abstract}
By means of inductive strategies that evoke the knowledge of boys and girls through the cultural syntax expressed in Jorge Gashé's (2008) Intercultural Inductive Method, this article presents the experience in the application of evocation strategies of indigenous knowledge related to fishing proven in children from a bilingual Ikoot primary school in San Mateo del Mar in the state of Oaxaca, Mexico. Some of the results are the approach made to the explanation and organization of indigenous knowledge, the approach to the topic of tacit observation as a valuable form of learning used in sociocultural diverse contexts.
\end{abstract}

Keywords: explicitation, indigenous knowledge, evocative strategies, education

\section{INTRODUCCIÓN}

La presente contribución es parte de una investigación realizada en una escuela primaria bilingüe perteneciente a la comunidad lacustre de San Mateo del Mar, ubicada en la región del Istmo de Tehuantepec, en el estado de Oaxaca, México. Se eligió por ser considerada 
por los mismos habitantes del lugar una comunidad predominantemente indígena, bilingüe (castellano y huave o ikoot) y donde aún persisten actividades y celebraciones que se consideran propias.

El objetivo original fue documentar y analizar etnográficamente las dimensiones institucionales, pedagógicas y socioculturales que intervienen y pueden favorecer la agencia docente en y para la diversidad cultural y la interculturalidad en la escuela primaria bilingüe Adolfo López Mateos, por conducto del Método Inductivo Intercultural (MII), así como de estrategias que visibilicen los aprendizajes socioculturales infantiles. Asimismo, apoyar la explicitación y sistematización de los conocimientos y valores implícitos en las actividades pesqueras como fuentes que pueden favorecer esta agencia docente. Este objetivo se logró a través de métodos y técnicas inductivas que incitaron a la transformación de la escuela "desde fuera"; es decir, con la participación de actores comunitarios que aportaron conocimientos ikoots y no "desde dentro", bajo una visión institucional que prioriza los conocimientos escolares, como se ha hecho generalmente.

En el caso de este trabajo, por cuestiones de extensión, se hará énfasis sólo en la dimensión sociocultural, en especial en el análisis de la experiencia acerca de la aplicación de estrategias inductivas evocadoras del conocimiento indígena en niños y niñas sobre el tema de la pesca y la manera en que estos saberes pueden ser organizados para su uso en el aula, a la par de los conocimientos escolares. Todos los niños colaboradores de esta investigación pertenecen a la comunidad de San Mateo del Mar y algunos de ellos también son estudiantes de la Escuela Primaria Bilingüe Adolfo López Mateos, de esa misma localidad.

Es importante aclarar que el MII implementado por Jorge Gasché, Jesica Martínez y Carmen Gallegos en la Amazonía peruana constituye un eje importante en esta investigación, ya que apoyó como una guía teórica y metodológica, porque se construye inductivamente (desde abajo), con la participación de las fuentes del conocimiento indígena: los alumnos, los docentes, los sabios de la comunidad y también por medio de las obras antropológicas.

El MII implica crear las condiciones de un diálogo más igualitario entre los actores escolares y los actores comunitarios, con el fin reco- 
nocer el valor del saber indígena y superar las relaciones de dominación y sumisión (Gasché, 2008b). Este método propone una visión sintáctica de la cultura, la que apoyó a tener una postura sobre el concepto; es decir, para esta investigación, la cultura es el resultado de las actividades humanas cotidianas, por ejemplo, la actividad pesquera. La cultura entendida así, con fines interculturales y a partir de una relación histórica conflictiva (Gasché, 2008b) y tensa como la que se ha tenido con la educación, puede llevar, finalmente, a una relación más o menos armónica por medio de la articulación de los conocimientos locales con los conocimientos escolares dentro y fuera del aula.

Para que dicha articulación ocurra, los conocimientos comunitarios pueden estar organizados de la misma manera que los conocimientos escolares; lo anterior se logró por medio de un acercamiento a las fuentes del conocimiento indígenas por medio de estrategias inductivas evocadoras del conocimiento y, posteriormente, se organizaron los conocimientos otorgados con herramientas como el Calendario Socionatural y la Matriz de Contenidos del Área Integradora Sociedad y Naturaleza (UNEM, 2009), que el mismo MII propone. ${ }^{1}$ Estos conocimientos organizados posibilitan generar con más facilidad propuestas pedagógicas que contribuyan a un proceso educativo que se construye desde las actividades comunitarias en las que participan los alumnos (Bertely, 2009) y que, en el caso de México, se ha extendido en distintas entidades de la república, incluido Oaxaca, por medio de la Red de Educación Inductiva Intercultural (Rediin). ${ }^{2}$

Tras señalar el objetivo general y dar un panorama general de lo que se pretende, el artículo explicita la metodología inductiva que permitió la aplicación de las estrategias en mención, para luego dar a

\footnotetext{
${ }^{1}$ El calendario Socionatural y la Matriz de Contenidos del Área Integradora Sociedad y Naturaleza se utilizaron en esta investigación como instrumentos que apoyaron la organización de los conocimientos comunitarios proporcionados por los diferentes actores, con el fin de facilitar su uso en el aula, junto con los conocimientos escolares.

${ }^{2}$ La Red de Educación Inductiva Intercultural (Rediin) se encuentra conformada por Nodos Primarios: UNEM y Educadores Independientes de Chiapas, así como por profesores-investigadores del CIESAS en convenio con el Instituto de Investigaciones de la Amazonía Peruana (IIAP). Nodos Secundarios: instituciones públicas que contribuyen al complimiento de los objetivos de la mencionada Red (UPN, DGEI, CEIB, las Universidades Interculturales y el INALI, entre otras), bajo diversas fuentes de financiamiento: Fondo Miguel León Portilla, Fundación Ford, Fundación Kellogs y, actualmente, la DGEI y el Conacyt, entre otras.
} 
conocer algunos resultados, conclusiones y recomendaciones acerca de la experiencia, pues permite tener datos que podrían contribuir a futuras investigaciones orientadas a una educación más incluyente.

\section{Objetivo}

El eje central de este documento es la identificación del conocimiento indígena explicitado por los niños por medio de estrategias evocadoras, a fin de establecer una relación entre éste y el conocimiento escolar; por lo tanto, el objetivo fue analizar la experiencia acerca de la aplicación de estrategias inductivas evocadoras del conocimiento indígena en nińos y nińas sobre el tema de la pesca y la posible organización de estos conocimientos para su articulación con los conocimientos escolares.

\section{Metodología}

En la investigación se utilizó un enfoque etnográfico que permitió hacer entrevistas y observaciones. A su vez, el MII se usó como una herramienta de análisis para probar la posibilidad de "atrapar" conocimientos escolares y comunitarios en torno a la actividad pesquera, sincronizando su potencial metodológico. Del mismo modo, permitió la colaboración de diferentes actores sociales y educativos de manera constructiva y creativa por medio de diversas estrategias inductivas evocadoras de los conocimientos de los niños y de las nińas acerca de la actividad pesquera como eje central. Es decir, por medio de la sintaxis cultural, que entiende la cultura como las actividades productivas que se realizan en la comunidad y la relación que guardan con los diferentes actores. De esta manera, la actividad es el punto de partida de los procesos pedagógicos interculturales y, a la vez, "el conocimiento participa funcionalmente como medio y toma su forma correspondiente a su función" (Gasché, 2008b, p. 279).

En este sentido, en la investigación en extenso se consideraron tres dimensiones de análisis: institucional, pedagógica y sociocultural. En este documento se centrará en el último con el fin de describir la explicitación por medio de la colaboración y el interaprendizaje de los conocimientos implícitos en las actividades pesqueras realizadas por los niños y niñas de la escuela primaria estudiada, al lado de sus familias, los comuneros y comuneras. Lo anterior con 
el fin de identificar su posible articulación en un currículo escolar interculturalizado.

\section{El concepto de cultura}

Se parte de una noción pragmática de la cultura que apoya la perspectiva inductiva y etnográfica, lo que inspiró este trabajo de investigación, así como de la sistematización de las pedagogías, los conocimientos y los valores indígenas y su presencia en las actividades comunitarias y en la institución escolar. Tomando en cuenta lo anterior, se hace necesario especificar que el concepto de cultura que se maneja en este documento, así como las tensiones entre normatividad e innovación docente en contextos interculturales es el que Jorge Gasché (2008b, pp. 279-280) define como:

el resultado observable de la actividad humana, y no como una serie de elementos y representaciones y valores simplemente disponibles y que, según algunos, determinan el actuar humano... la cultura considera como resultado de la actividad no es entonces una entidad estable, sino evolutiva cuya realización y aspecto concreto depende de las opciones que sus miembros toman en cada instante de su actuar. De este concepto de una cultura producida y condicionada por la actividad de las personas se deriva, desde luego, una estrategia pedagógica intercultural que parte de las actividades productoras de cultura tales como se practican y se las puede observar en la vida de una comunidad indígena.

Si se considera que la cultura es resultado de la actividad humana, las prácticas pedagógicas interculturales podrían tener significado más cercano a la vivencia de los diferentes actores y en especial de los niños en el aula. Según Gasché, además de una pedagogía bilingüe, la escuela debería tomar en cuenta la herencia cultural de las comunidades y fomentar el desarrollo del niño a partir de su universo cultural y lingüístico local; sólo así se puede construir una escuela socioculturalmente pertinente. Lo anterior necesita de una reflexión teórica en el medio indígena bajo "una estrategia conceptual y expositiva adecuada y adaptada al universo lingüístico, cognitivo y cultural de los interlocutores indígenas y no puede limitarse al uso 
de los manuales universitarios comunes y corrientes en el medio urbano" (Gasché, 2008b, p. 304). Para lograr lo anterior, se requieren formadores que tengan claro el universo cultural, cognitivo y lingüístico al cual deben adaptar su discurso pedagógico y que significa el primer recurso a partir del cual se puede desarrollar el MII.

\section{El conocimiento indígena en el niño}

En la investigación se realizaron charlas con diferentes actores, a quienes podemos clasificar como comunitarios - padres y madres de familias de pescadores, algunos de sus hijos y docentes conocedores de la actividad-y escolares -docentes y alumnos-. Recordemos que, desde el punto de vista de Gasché, son las cuatro fuentes del conocimiento indígena; para este trabajo de investigación, todos ellos son, en alguna medida, comunitarios. La primera fuente del conocimiento indígena está en el nińo, la segunda en el maestro, la tercera en los sabios de la comunidad y la cuarta en las obras antropológicas. Dado el objetivo del presente artículo, la atención se centrará en el niño como una de las fuentes del conocimiento indígena.

Es importante reconocer que el niño ingresa a la escuela y trae consigo una cultura adquirida en la socialización primaria, que incluye conocimientos familiares y culturales aprendidos en su vida en comunidad. De esta manera, el nińo "tiene" conocimientos previos que el docente debe ampliar y articular interculturalmente en el aula; es decir, se necesita que el docente desarrolle habilidades $\mathrm{y}$ actitudes que faciliten el trabajo pedagógico dentro y fuera del aula de acuerdo con las etapas del desarrollo cultural por las que transitan sus alumnos y alumnas, ycon los conocimientos, valores y significados familiares y culturales que podrían articularse o contrastarse con los contenidos escolares.

En cuanto a la adquisición del conocimiento en el niño, merece bien la pena reconocer el aporte del concepto de Zona de Desarrollo Próximo (ZDP) acuñado por Vygotsky (1988) que, a su vez, utiliza el concepto de interaprendizaje como andamiaje para la construcción del conocimiento indígena y cultural del niño. La ZDP se define como "la distancia entre el nivel real de desarrollo, determinado por la capacidad de resolver independientemente un problema, y el nivel de desarrollo potencial, determinado a través 
de la resolución de un problema bajo la guía de un adulto o en colaboración con otro compañero más capaz" (Vygotsky, 1988, p. 133), por lo que el docente debe poseer la habilidad de "conocer" los conocimientos previos del estudiante y planear la manera en que éstos se podrían ampliar con los conocimientos escolares, en un mismo plano de importancia.

Se debe reconocer que el niño aprende o interaprende en su comunidad, a partir de su involucramiento práctico en las actividades familiares y comunitarias, con el apoyo de adultos o pares más capaces, por lo que la interacción entre el niño y el contexto comunitario (entendido social y culturalmente) desempeña un papel esencial. El niño es un ser cultural, así que el conocimiento se construye a través de la interacción social mediada por la cultura, de ahí la importancia de que el docente conozca acerca de los principales elementos culturales que identifican a la comunidad en cuestión, con el fin de facilitar el proceso de identificación de los conocimientos previos que traen consigo los nińos como fuentes del conocimiento indígena.

Es menester reconocer el proceso de separación de las actividades comunitarias que tiene el niño a partir de su incorporación a las actividades escolares; dicho de otra manera, el hecho de asistir a la escuela trae como consecuencia que los niños no participen de igual manera en las actividades comunitarias y familiares como la pesca; no obstante, los primeros años de edad son fundamentales para la adquisición de los conocimientos que les servirán para su vida futura en la comunidad.

En este sentido, en las diversas entrevistas realizadas a los padres de familia que colaboraron en esta investigación, éstos expresaron la importancia que para ellos representa enviar a sus hijos a la escuela, porque no desean que éstos últimos pasen lo que ellos han pasado por no tener una formación escolarizada, por lo que refirieron no tener interés en enseñar la actividad pesquera a sus hijos, además de que representa una actividad pesada y riesgosa. Mencionaron también que no perciben interés en participar en la pesca por parte de los niños, ya que solamente se dedican a jugar y esta actividad requiere responsabilidad y disciplina. Los padres de familia consideran que la "escuela" es lo más importante en el desarrollo del nińo, por lo que esa debe ser su actividad primordial "para ser alguien en la vida”. 


\section{ESTRATEGIAS DE APROXIMACIÓN AL CONOCIMIENTO INDÍGENA EN EL NIÑO}

Para el proceso de investigación, las aseveraciones de los padres cerraban las posibilidades de que los hijos tuviesen conocimientos acerca de la actividad pesquera. Sin embargo, a manera de comprobación, se ideó una estrategia de aproximación con los niños y niñas para percatarnos de la existencia o no de dichos conocimientos.

Se construyó una estrategia inductiva evocadora de los conocimientos de los niños y de las niñas a través del dibujo, con consignas que se derivan de los aspectos considerados en la sintaxis cultural. Esta estrategia detonó, a partir de reuniones con los niños donde se realizaron charlas, que expresaran de manera verbal sus conocimientos previos sobre la actividad pesquera. El primer paso fue establecer algunas preguntas guías para las pláticas y actividades a realizar con base en la frase prototípica que atrapa el enfoque sintáctico de cultura: "los hombres van al territorio a obtener recursos que transforman con determinadas técnicas e instrumentos para satisfacer necesidades productivas, laborales, sociales o ceremoniales" (Gasché, 2008a, p, 157). Las preguntas que se hicieron se relacionaron con el territorio (¿Dónde se realiza la actividad?), con los recursos (¿Con qué realizas la actividad?), con los procesos técnicos, instrumentales y de transformación (¿Cómo y con qué realizas la actividad?) y con el fin social de la actividad (¿para qué la realizas?). En la plática, se les solicitó que dibujaran lo que comentaban, y esto dio como resultado que el dibujo se convirtiera en una estrategia evocadora de los conocimientos implícitos en la actividad pesquera, ya que ayudó a explicitarlos y a profundizar en ellos.

\section{¿EL LUGAR IMPORTA? EN EL CONTEXTO COMUNITARIO O EN LA ESCUELA}

\section{La experiencia en la casa}

Es importante recalcar que las charlas y las reuniones grupales con los niños se realizaron en dos contextos totalmente diferentes: en casa y en la escuela. En el primero se dio durante las vacaciones 
de fin de año escolar, siendo una experiencia reconfortante ya que, dadas las fechas en que se realizaron, se contó con participantes no contemplados en un primer momento, pero cuyas aportaciones enriquecieron los datos. Las edades de los niños oscilaban entre los cinco y los siete ańos de edad.

Cuando los niños se sintieron en confianza para participar, lo que se logró fueron charlas en las que ellos debatían aspectos relacionados con la pesca, por ejemplo: lugares donde se practica la actividad, tipo de productos pesqueros, características de los productos pesqueros, técnicas de pesca, descripción de los instrumentos de pesca y fines sociales de los productos, lo cual lleva implícita la sintaxis cultural. Realizaban este debate mientras dibujaban lo que describían, por lo que el dibujo tomó el papel de evocador para que los nińos explicitaran los conocimientos relacionados con mencionada actividad.

En el curso de la técnica, los padres de familia también se mostraron colaborativos, complementando información cuando los niños dudaban al describir algún suceso o instrumento, lo cual mostró el valor al interaprendizaje como otra característica del MII. Dada la eventual ayuda de los padres, fueron los niños quienes discutieron los temas, logrando un debate interesante sobre la actividad pesquera. Finalmente, se pudo notar que los padres de familia se mostraban sorprendidos por la cantidad de conocimientos que los niños expresaban acerca de una actividad que no realizaban y hacia la que no manifestaban interés.

El uso de la técnica del dibujo fue sorprendente y alentador con respecto al trabajo de investigación con las niñas y niños, quienes mostraron que poseen un conocimiento fragmentado pero vasto de la actividad. No obstante, durante la investigación se constató -como lo mencionaron los padres de familia- que ellas y ellos no participan de manera directa en la actividad. Sin embargo, el hallazgo fue que, lo que conocen, lo han aprendido por medio de la observación como una forma de aprendizaje sociocultural que los niños han adquirido al participar como observadores en las actividades que se realizan en su contexto familiar y social y por la frecuencia con la que viven, precisamente, diversas actividades relacionadas con la actividad pesquera. 


\section{LA EXPERIENCIA EN LA ESCUELA}

El otro escenario en el que se llevó acabo la actividad fue la escuela. De forma previa a la actividad, se platicó con el director -en primera instancia- y con los docentes -más tarde- sobre las actividades planeadas con los grupos, los tiempos de duración de las mismas y los objetivos trazados; finalmente, se solicitó autorización para realizarlas. Se llevaron a cabo reuniones con los grupos de $2^{\circ}, 4^{\circ}$ y $6^{\circ}$ grados. La dinámica fue la siguiente: se ingresó al salón de clases y el docente de $6^{\circ}$ grado se retiró del aula; no fue así en el caso de los docentes de $2^{\circ}$ y $4^{\circ}$ grados. El dato anterior se vuelve importante, porque la presencia o no de los docentes permitió que se desarrollaran dinámicas diferentes en la participación de los niños al responder las preguntas guía hechas por la investigadora. En el caso del $2^{\circ}$ grado, aunque la docente permaneció en el aula, los alumnos se mostraron muy participativos, abiertos y sabedores de la actividad pesquera.

Por otro lado, los alumnos de $4^{\circ}$ grado se mostraron participativos, pero temerosos: con mucha frecuencia volteaban a ver al docente como si éste supiera la respuesta a las preguntas que la investigadora proponía y con la mirada hacia el docente buscaban la aprobación de lo dicho. Esto parecía sugerir que la presencia del docente no implica, necesariamente, el silenciamiento de los conocimientos no escolares de los niños y las niñas, que suelen no formar parte de las clases regulares, pero que en un espacio experimental como el generado pueden surgir inhibiciones dependiendo hipotéticamente de la confianza establecida en la relación docente-alumnos. Además de esta relación de confianza, el caso de los alumnos de $6^{\circ}$ grado planteó la posibilidad de otra interpretación. Aunque no se contó con la presencia del docente, ellos y ellas no proporcionaron muchos datos, se mostraron apenados y temerosos al responder. Si comparamos el comportamiento entre el alumnado de $2^{\circ}, 4^{\circ}$ y $6^{\circ}$, sus respuestas parecen objetivar el papel aculturador ${ }^{3}$ de la escuela cuando, a menor ex-

\footnotetext{
${ }^{3}$ Para Aguirre (1957), la aculturación se refiere al contacto de culturas, es decir, la transmisión y asimilación cultural de las influencias recíprocas que se dan entre las culturas y usualmente se da en detrimento de las bases socioculturales (lengua, tradiciones, costumbres, conocimientos, significados y valores) de una de ellas.
} 
posición escolar, corresponde mayor confianza para hablar de las actividades y conocimientos locales y, a mayor exposición menos confianza, vergüenza y temor a manifestarlos.

El papel de la investigadora fue de guía haciendo preguntas para que los niños explicitaran los conocimientos relacionados con la pesca. Las preguntas no fueron lineales, tomaron dirección con base en el interés de los niños. Cabe la pena mencionar que dado el éxito de la actividad evocadora hecha por medio de los dibujos, se entrevistó a los nińos en sus casas, se determinó repetir la actividad, por lo que los niños con los que se realizaron las reuniones grupales también dibujaron mientras describían diversos aspectos relacionados con la pesca. Todo lo anterior fue videograbado para su sistematización y análisis.

Recordemos que, en la investigación que da como resultado este artículo, se entrevistó a colaboradores que se clasificaron como comunitarios y escolares. En este sentido, todos estos colaboradores son considerados fuentes del conocimiento indígena y, para poder "tener" los conocimientos comunitarios y facilitar su uso pedagógico en la escuela, es necesario un acercamiento a este tipo de actores.

En la investigación se visitó a las familias en sus hogares, existió colaboración en algunas actividades relacionadas con la pesca y, de la misma manera, existieron visitas en la escuela en mención. Dicha estrategia permitió que los datos sobre los conocimientos indígenas fueran el resultado de una descripción mucho más densa que la que puede arrojar una entrevista convencional. La actividad fue central para la identificación de los conocimientos que cada uno de ellos aportó.

\section{Algunos conocimientos explicitados. ¿Cómo los organizo?}

Para organizar los conocimientos explicitados por los nińos, lo mismo que su posible articulación y ampliación intercultural, se utilizó como herramienta la Matriz de Contenidos del Área Integradora Sociedad y Naturaleza; esta matriz, menciona Gasché (2008b, 170), opera como una "red para pescar contenidos de las actividades sociales” por medio del MII, ya que los conocimientos indígenas -que son dinámicos y cambiantes- no se encuentran de la misma manera que los conocimientos escolares-universales. La idea fue utilizar esta 
matriz con el fin de aprovechar pedagógicamente las actividades comunitarias -en este caso, las relacionadas con la pesca-, así como su posible articulación y ampliación intercultural (UNEM, 2009, p. 85). Esta sistematización facilita su uso en prácticas pedagógicas interculturales y en la gestión docente en y para la diversidad cultural.

Los niños comenzaron describiendo algunos de los productos más comunes de la actividad pesquera: El tixem o camarón, su pesca se lleva a cabo en el mar muerto. Los nińos discutieron acerca de las principales características de este producto, por ejemplo: características físicas, localización del producto, instrumento que se utiliza para su pesca, clasificación del producto para la venta, costos del producto y lugares de venta:

Areli: ¿Camina el camarón?

María Luisa: No sé, ¿`camina?

Areli: Sí, camina porque tiene pies.

Carlitos: No, abajo no camina, camina en el agua, mueve sus pies.

Areli: $\quad$ Cuando está abajo del mar, en la tierra no camina. Algunos brincan.

Leonardo: Hay unos grandes que tienen pinzas y tienen unas cosas como hilos, pero no son hilos, le sale de los ojos, es como pelos.

Jesús: $\quad$ Mi papá atrapó uno bien grande, con copo, y lo vendió mi mamá en el mercado o si no, lo vende en Salina Cruz, si es mucho camarón, va a Salinas.

María Luisa: ...Y, ¿a cómo está el camarón?

Areli: $\quad$ Pues, por bolsas, algunas te venden llena, algunos son de chiquitos, algunos son de grandes, los venden aparte. Los grandes cuesta mucho y los chiquitos cuestan menos.

Como se puede leer, los niños comenzaron a discutir algunos aspectos sobre los camarones, mientras realizaban algunos dibujos sobre el producto en cuestión: 


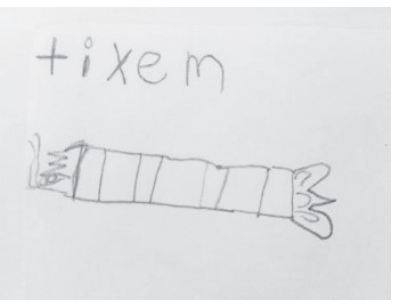

Fuente: registro en campo.

De repente, llegó el papá de Carlos y ayudó a dibujar un camarón. Él comentó que el camarón tiene 6 patas delanteras y 6 patas traseras:

- Imagen 2. Dibujo de Tixem hecho por el papá de Carlos

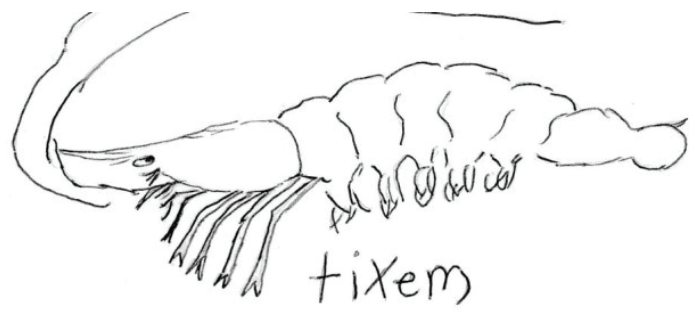

Fuente: registro en campo.

Después siguió la discusión sobre el Tixem, en este caso, se referían al papá de Carlos, preguntándole los datos que habían discutido anteriormente; se obtuvieron nuevos datos sobre las características físicas del camarón y la forma en la que se comporta en el mar:

Areli: ¿Verdad que el camarón camina?

Sr. Carlos: Sí, camina, pero...

Carlitos: En la tierra no camina, ¿verdad?

Sr. Carlos: Sí, camina en la arena, pero camina así (muestra con señas), despacito, y en el mar camina más rápido y se mueve en forma circular, cuando se quiere escapar es en forma de círculo, no en forma de línea, se corre rápido y a veces salta. 
Leonardo: Éste es pen, es un camarón también pero el pen es más chiquito, es del mar vivo y el tixem vive en el mar muerto.

La experiencia anterior ayuda a analizar el interaprendizaje que se da entre los niños y el Sr. Carlos, quienes interactúan para complementar o corregir información acerca de la pesca. Según Gasché, el interaprendizaje es la "palabra clave que evoca el proceso fundador de todo proyecto educativo intercultural" (Gasché, 2008a; 2008b, p. 384).

Posteriormente, el señor se retiró porque venía de pescar, situación que se aprovechó y se extrajo del canasto una jaiba que el Sr. Carlos había obtenido:

Areli: $\quad$ Ésta es una jaiba, se parece a los cangrejos, pero los cangrejos viven en el mar vivo, debajo de la tierra. Saben sabroso, en mi casa lo comemos.

Carlitos: Estas jaibas tienen dos tenazas, sirve para morder. María Luisa: ¿Y cuántas patas tiene?

Carlitos: Cuatro y se atrapa con tarraya en el mar muerto, la jaiba en el mar vivo, ah no, no tiene cuatro, ya lo conté, tiene diez patas, cuatro de un lado, cuatro del otro y dos en su cabecita.

Areli: $\quad$ Las jaibas y los cangrejos caminan de lado, quién sabe por qué.

A continuación, se presentan dos dibujos de las jaibas hechos por los niños.

- Imagen 3. Dibujos de la jaiba hecho por dos de los niños
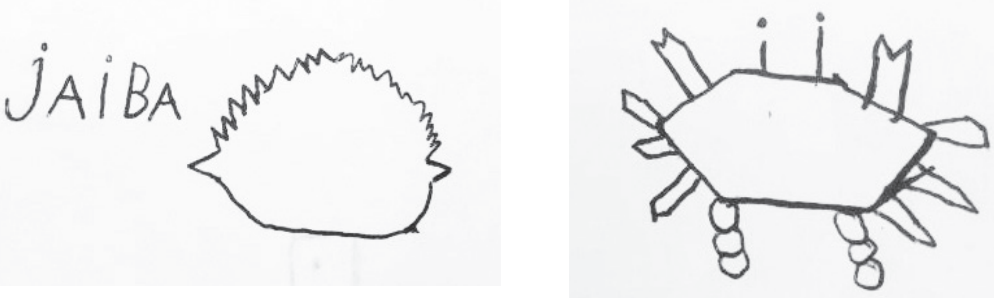
Posteriormente, el Sr. Carlos acercó a la mesa una libreta donde tenía dibujos hechos por él. Comentó que fue un trabajo que la maestra de primer año había pedido a los padres de familia. Con base en esos dibujos, los niños continuaron hablando de otros tipos de productos, como el pulpo y otro tipo de camarón, el tiburón, el pez sierra y el calamar:

Carlitos: Éste es un pulpo, vive en el mar vivo y en el mar muerto y se atrapa con tarraya.

Areli: $\quad$ Ése se coce como se coce el camarón, se hace caldo.

Carlitos: Nosotros lo comemos como ensalada.

Areli: $\quad$ No se hace ensalada.

Carlitos: Sí se hace, pregúntale a mi mamá vas a ver.

Leonardo: Mira, Éste se llama pen, vive en el mar vivo y el tixem vive en el mar muerto, el pen o chacal es más chiquito y vive en la laguna.

Areli: $\quad$ Tienen una puntita en su trompita, ¡qué lástima!

Leonardo: Mira, también hay tiburón y vive en el mar vivo, pero ése no se pesca. Lo pescan barcos más grandes y lo venden lejos.

María Luisa: ¿Qué otra cosa?

Carlitos: El pez sierra, se atrapa con tarraya.

María Luisa: ¿Se come?

Carlitos y Areli: No

Carlitos: Ése mata gente, corta con su sierra, vive en el mar vivo, nosotros no lo comemos, pero otra gente sí.

Areli: Hay un calamar.

Carlitos: Yo lo he visto (llama a su papá para que dibuje un calamar) mi papá los atrapa y sabe bien rico, también lo vende mi mamá. 
- Imagen 4. Dibujo del calamar hecho por el papá de Carlos

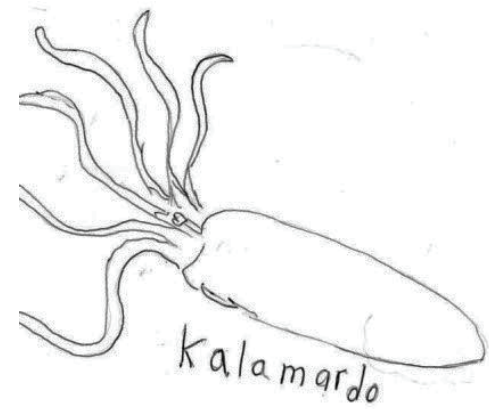

Fuente: registro en campo.

También describieron algunos de los instrumentos mencionados durante la actividad: tarraya y kopo.

- Imagen 5. Dibujos de la tarraya o dok hecho por los niños como otro instrumento de pesca utilizado principalmente en el mar vivo.

Fuente: registro en campo.
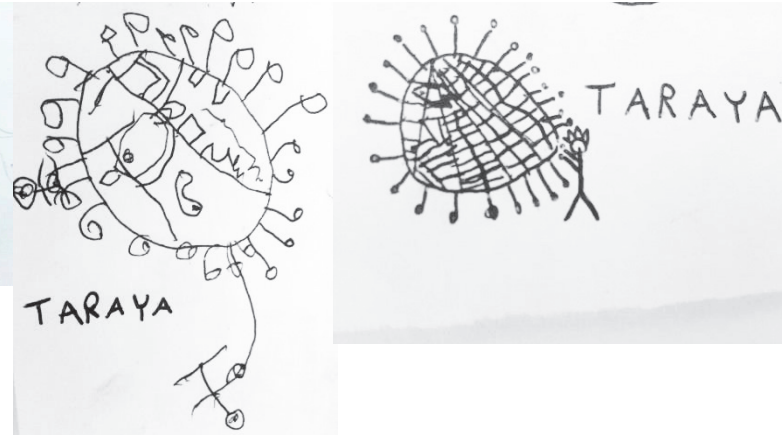

Areli: $\quad$ La tarraya se avienta.

María Luisa: ¿Y dónde se utiliza la tarraya?

Areli: $\quad$ En el mar vivo.

Sr. Carlos: Pero también en el mar muerto y en la laguna, se avienta.

Carlitos: Con eso se atrapa jaiba, pescados y charales.

A continuación, se presenta una imagen con dibujos de un instrumento de pesca utilizado en el mar muerto para la pesca de camarones, principalmente. 

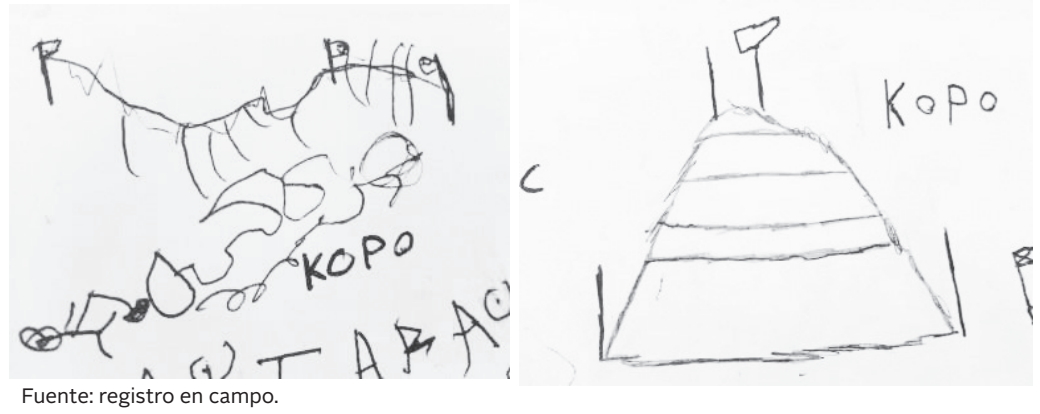

Leonardo: El kopo sirve para atrapar camarón.

María Luisa: ¿En dónde se utiliza?

Leonardo y Carlitos: En el mar muerto.

Leonardo: Se amarra de tres palos, se tiene que bajar mi papá a amarrarlo, nada y amarra con unos mecates en los tres palos.

María Luisa: ¿A qué hora se hace?

Carlitos: En la noche, porque los camarones salen en las noches.

Sr. Carlos: Se utiliza lámparas para llamar a los camarones y se ponen tres o cuatro kopos, dependiendo del número de kopos son los palos.

María Luisa: ¿Y se pesca mucho así?

Carlitos: Más cuando está nublado o más obscuro.

Sr. Carlos: Cuando hay luna llena, se pesca menos, cuando está nublado se pesca más.

Con base en los conocimientos presentados y los datos proporcionados por los niños, se muestra un ejemplo de la Matriz de contenidos del Área Integradora Sociedad y Naturaleza relacionados con la pesca realizada. 
Cuadro 1. Matriz de contenidos del Área Integradora Sociedad y Naturaleza relacionados con la pesca realizada con base en datos proporcionados por los niños

\begin{tabular}{|c|c|c|c|}
\hline Territorio-naturaleza & Recurso natural producto & Trabajo-técnica & Fin social-sociedad \\
\hline \multirow{3}{*}{ Mar muerto } & Tixem (camarón) & Kopo & $\begin{array}{l}\text {-Venta en el mercado- } \\
\text { Consumo familiar }\end{array}$ \\
\hline & Jaiba & Tarraya & $\begin{array}{l}\text {-Venta en el mercado } \\
\text {-Consumo familiar }\end{array}$ \\
\hline & Pulpo & Tarraya & -Consumo familiar \\
\hline Laguna & Pen o chacal & Tarraya & $\begin{array}{c}\text {-Venta } \\
\text {-Consumo familiar }\end{array}$ \\
\hline \multirow{3}{*}{ Mar vivo } & Pulpo & \multirow{3}{*}{ Tarraya } & -Consumo familiar \\
\hline & Tiburón & & -Venta \\
\hline & Pez sierra & & -Venta \\
\hline
\end{tabular}

Fuente: elaboración propia.

Complementando el cuadro anterior, se presenta la matriz que integra los datos proporcionados por los niños de $2^{\circ}, 4^{\circ}$ y $6^{\circ}$ grados con los cuales se realizaron las reuniones grupales dentro de la escuela.

Además de los conocimientos mencionados en las matrices que se presentaron, en general, los niños referenciaron temas como los roles de género en la actividad y los principales platillos con los productos de la pesca.

Como se puede observar, el conocimiento indígena se aprecia en distintas fuentes: niños, comuneros, docentes y escritos antropológicos y, según Gasché (2008b, pp. 306-307):

aun cuando el conocimiento indígena exige un esfuerzo previo a su uso pedagógico: el de objetivarlo notándolo por escrito, inventariándolo, sistematizándolo e integrándolo en manuales y materiales escolares... es decir... convertirlo en un saber que existe en las mismas condiciones materiales que el saber escolar convencional y que, desde luego, puede ser manejado de la misma manera en el aula... de una existencia exclusivamente oral y arraigada en las mentes y los gestos, el conocimiento indígena, al parecer debe adquirir una existencia escrita para que sea conservado en libros y, de esa manera, alcance el mismo estatus material y operativo que el conocimiento escolar convencional. 
Cuadro 2. Matriz de contenidos del Área Integradora Sociedad y Naturaleza relacionados con la pesca realizada con base en datos proporcionados por los niños de la Escuela Primaria Bilingüe Adolfo López Mateos

\begin{tabular}{|c|c|c|c|}
\hline Territorio-naturaleza & $\begin{array}{c}\text { Recurso } \\
\text { naturalproducto }\end{array}$ & Trabajo-técnica & Fin social-sociedad \\
\hline \multirow{3}{*}{$\begin{array}{r}\text { Mar muerto } \\
\text {-Hay palos para } \\
\text { amarrar la tarraya y } \\
\text { el copo } \\
\text {-No hay olas }\end{array}$} & Tixem (camarón) & Kopo & $\begin{array}{l}\text {-Venta en el mercado } \\
\text {-Consumo familiar } \\
\text { (caldo de camarón) }\end{array}$ \\
\hline & Jaiba & Tarraya & $\begin{array}{l}\text {-Venta en el mercado } \\
\text {-Consumo familiar } \\
\text { (hervido con sal) }\end{array}$ \\
\hline & Pulpo & Tarraya & $\begin{array}{c}\text {-Consumo familiar } \\
\text { (ensalada) }\end{array}$ \\
\hline $\begin{array}{r}\text { Laguna } \\
\text {-No hay olas }\end{array}$ & Camarón & Tarraya & $\begin{array}{c}\text {-Venta } \\
\text {-Consumo familiar }\end{array}$ \\
\hline \multirow{4}{*}{$\begin{array}{l}\text { Mar vivo } \\
\text {-No hay palos } \\
\text {-Olas grandes }\end{array}$} & Pulpo & \multirow{3}{*}{ Tarraya } & -Consumo familiar \\
\hline & Pescado & & $\begin{array}{c}\text {-Venta } \\
\text {-Consumo familiar (pescado frito y } \\
\text { caldo de camarón) }\end{array}$ \\
\hline & Medusas & & \\
\hline & Huevo de tortuga & $\begin{array}{l}\text { Recolección en la } \\
\text { playa }\end{array}$ & -Venta \\
\hline
\end{tabular}

Fuente: elaboración propia.

La realidad es que son escasos los materiales pedagógicos que faciliten el diálogo de saberes (De Sousa, 2006) entre el conocimiento escolar y los conocimientos indígenas en un mismo nivel de importancia. En este sentido, y para minimizar la desventaja mencionada y con base en el MII, en este trabajo se plantea un primer esfuerzo por explicitar el conocimiento indígena relacionado con la pesca, con el fin de que el docente descubra de manera paulatina una alternativa que le permite partir del conocimiento indígena para apoyar los procesos pedagógicos y desarrollar competencias interculturales y favorecer a la agencia docente en y para la diversidad cultural.

No existen recetas para la atención de la diversidad cultural en el aula, sino sugerencias. El MII sugiere que el docente apoye la 
explicitación de los conocimientos comunitarios, su profundización y organización curricular por medio de los cuatro ejes que estructuran el enfoque sintáctico de cultura y, por tanto, la Matriz de Contenidos y el Calendario. Los cuatro ejes son: objeto/recurso natural (¿qué?), medio ambiente/naturaleza (¿dónde?), transformación/técnica (¿con qué?, ¿cómo?), fin social/sociedad (¿para qué?). Estos ejes facilitan la articulación intercultural de los conocimientos comunitarios y escolares mediante un proceso que atiende lo genérico a través de lo específico, con el fin de explicitar los conocimientos implícitos en la acción. Con base en estos ejes, Gasché (2008a, p. 315) propone una oración que expresa de manera concreta, pero genérica, en qué consiste lo que se llama una actividad productora y actualizadora de cultura y conocimiento indígena: "el ser humano produce cosas yendo a la naturaleza a sacar sus materiales para transformarlos con el fin de satisfacer una necesidad (de forma social)".

\section{CONCLUSIONES}

Como se ha mencionado en el apartado metodológico, se utilizaron estrategias inductivas evocadoras de los conocimientos de los niños y de las niñas a través de la sintaxis cultural. Ésta se constituye por estrategias que surgieron en la marcha y se planearon para comprobar la existencia o no de una relación directa entre la actividad pesquera y los conocimientos que los niños tienen sobre la mencionada actividad. Esto por medio de charlas sustentadas en preguntas guiadas y coloquiales que correspondieron al concepto sintáctico de cultura y que fueron complementadas con dibujos que los niños hicieron acerca de territorio, productos, técnicas y fines sociales de la actividad pesquera. Estas estrategias se constituyeron en una herramienta muy útil para que, finalmente, el niño explicitara conocimientos comunitarios relacionados con la pesca.

\section{¿Qué pasa si la actividad está ausente en los niños?}

La utilización de estas estrategias contribuyó al MII (Gasché, 2008b), ya que este método tiene su punto de partida en el concepto sintáctico de cultura, es decir, entiende que la cultura es resultado de la actividad; 
por lo tanto, parte de la actividad como centro pero, ¿qué implicaciones tiene si los niños no participan directamente en la actividad pesquera, como mencionan los padres de familia? Esta pregunta surge a partir del acercamiento a la cultura ikoot y, específicamente, a la actividad pesquera, ya que los padres de familia reportaron que no existía una participación directa de los niños en dicha actividad. Sin embargo, en esta investigación se pudo comprobar que la ausencia de participación en la actividad pesquera no imposibilita que los niños tengan conocimientos acerca de la misma. Es importante resaltar que la actividad constituye un proceso fundamental para la activación de las fuentes del conocimiento indígena; es decir, a partir de la aproximación a la actividad pesquera se puede lograr la explicitación de acciones específicas y más cercanas a la realidad. Este acercamiento trae consigo el análisis de los pasos de la acción y la reflexión sobre los conocimientos implícitos.

Finalmente, se constató que, a través de las estrategias mencionadas, se pudieron explicitar los conocimientos relacionados con la actividad pesquera sin necesidad de la participación de los nińos en la actividad mencionada, lo anterior no minimiza la importancia de la actividad como el principal motor de aprendizaje y transmisión cultural, sino que respeta la concepción de los padres acerca de las necesidades físicas del niño y el nivel de seriedad y responsabilidad que requiere la actividad pesquera.

\section{La importancia de la alfabetización territorial: la alfabetización tácita}

La observación tácita en torno a la actividad pesquera que se practica en la comunidad ikoot se convierte en una forma de aprendizaje valiosa que es parte de la alfabetización territorial (Bertely, 2007b); es decir, que la observación representa una habilidad utilizada en contextos socioculturalmente diversos (Bertely, 2000; Podestá, 2011 y Paradise, 1991). De este modo, las estrategias inductivas evocadoras de los conocimientos de los nińos y de las nińas sobre la pesca permitieron la explicitación de los conocimientos implícitos en la actividad, que los niños adquirieron gracias a la observación; en consecuencia, la participación de los niños no solamente se da en el hacer, sino por medio de la observación. 
La concepción de currículo de primaria del MII se desarrolla pensando que las actividades pedagógicas se dan a partir de una actividad social, la cual se va desagregando en otras actividades y de manera transversal llevan al tratamiento de conocimientos propios y escolares. La planeación no debe ser estática sino todo lo contrario; el docente puede identificar diversos conocimientos en términos interculturales para producir una planeación dinámica y proponer situaciones didácticas áulicas y extra áulicas in situ que ayuden a la articulación de los conocimientos escolares y los comunitarios. Además, debe considerar la necesidad de secuenciar los aprendizajes en función del grado escolar y las etapas del desarrollo cultural de las niñas y los niños e involucrarlos en la actividad por conducto de la acción. En términos pedagógicos, el docente debe planear y gestionar los procesos para que los niños puedan observar y hacer la actividad en cuestión. Esto traerá consigo conocimientos significativos, el interés de los niños y nińas por investigar al observar o hacer la actividad, el reconocimiento del conocimiento comunitario y el fortalecimiento de la identidad ikoot.

En este trabajo se comprueba que la alfabetización territorial (Bertely, 2007b) puede darse en contextos socioculturalmente diversos; esto por conducto de la actividad o, en su defecto, a través del aprendizaje observacional. En la presente investigación, las estrategias inductivas evocadoras de los conocimientos de los niños y de las niñas sobre la pesca a través de charlas y dibujos derivados de la sintaxis cultural funcionaron de manera extraordinaria, toda vez que permitieron la explicitación de conocimientos implícitos en la actividad que los niños adquirieron gracias a la observación. Por medio del Calendario Socionatural y la Matriz de Contenidos del Área Integradora Sociedad y Naturaleza se organizaron los conocimientos que pueden permitir su interculturalización dentro del aula.

Según Vygotsky, "el desarrollo cognitivo del niño es gracias a la interacción con otros actores comunitarios con más experiencia o expertos en las prácticas comunitarias y son ellos lo que también podrían mediar la actividad intelectual" (citado en Rogoff, 1997, p. 111). Como se pudo observar, los niños poseen un cúmulo de conocimientos que no fueron adquiridos por medio de la enseñanza directa de sus padres, sino por medio de la observación y de su interacción con el contexto comunitario y, por lo tanto, con la actividad 
pesquera. Según Rogoff (1997, p. 111), "los niños toman parte en las actividades de su comunidad involucrándose con otros niños o adultos en procesos rutinarios y tácitos, o también explícitos, de colaboración y que a partir de tal proceso de participación se preparan para su ulterior participación en situaciones semejantes". Así es como las nińas y los niños ikoots participan en los procesos de aprendizaje implícitos en las actividades comunitarias. Han adquirido los conocimientos comunitarios relacionados con la actividad pesquera por medio de la observación e interacción social y, en un segundo momento, cuando sean mayores y se encuentren físicamente preparados para realizar la actividad, pondrán en práctica todos estos conocimientos. Pero sí participan en actividades derivadas de la pesca que, de hecho, mostraron su potencial pedagógico.

Los niños colaboradores de esta investigación observan y se encuentran en proceso de aprendizaje de las actividades comunitarias relacionadas con la actividad pesquera; algunos de estos nińos saben el proceso de limpia y selección del camarón, pero no realizan dicha actividad aún. Por su parte, la experiencia y conocimiento de los niños que sí realizan la actividad y comparten un mismo grupo ayudará a ampliar el conocimiento de quienes no lo hacen. Según Vygotsky, "el desarrollo cognitivo del niño es gracias a la interacción con otros actores comunitarios con más experiencia o expertos en las prácticas comunitarias y son ellos lo que también podrían mediar la actividad intelectual” (citado en Rogoff, 1997, p. 111). Por lo tanto, esta planeación puede funcionar tanto con los niños que realizan la actividad, como con los que no la realizan, pero que, gracias al aprendizaje observacional, tienen conocimientos sobre la actividad pesquera adquiridos en la vida cotidiana por medio de la experiencia.

\section{REFERENCIAS BIBLIOGRÁFICAS}

Aguirre, G. (1957). El proceso de aculturación. México: UNAM.

Bertely, M. (2009). Innovación curricular en dos organizaciones indígenas de Chiapas. En R. López, J. A. Serrano, M. Bertely, S. Schmelkes e I. Álvarez. Cultivar la innovación: hacia una cultura de la innovación (pp. 19-63). México: COMIE. SEP.

Bertely, M. (2007). Conociendo a nuestras escuelas. Un acercamiento etnográfico a la cultura escolar. México: Paidós. 
Bertely, M. (2007b). Conflicto intercultural, educación y democracia activa en México. Cuidadanía y derechos indígenas en el movimiento pedagógico intercultural bilingüe en Los Altos, la Región Norte y la Selva Lacandona de Chiapas. México: CIESAS/Fondo editorial de la Universidad Católica del Perú.

Bertely, M. (2000). Familias y niños mazahuas en una escuela primaria mexiquense. Etnografía para maestros. México: Horizontes Alternativos.

De Sousa, B. (2006). La Sociología de las Ausencias y la Sociología de las Emergencias: para una ecología de saberes. En Renovar la teoría crítica y reinventar la emancipación social (pp. 13-41). Buenos Aires: CLACSO.

Gasché, J. (2008a). La motivación política de la educación intercultural indígena y sus exigencias pedagógicas. ¿Hasta dónde abarca la interculturalidad? En M. Bertely, J. Gasché y R. Podestá (coords.), Educando en la Diversidad. Investigaciones y experiencias educativas interculturales y bilingües (pp. 367-400). Quito, Ecuador: Abya-Yala.

Gasché, J. (2008b). Niños, maestros, comuneros y escritos antropológicos como fuentes de contenidos indígenas escolares y la actividad como punto de partida de los procesos pedagógicos interculturales: un modelo sintáctico de cultura. En M. Bertely, J. Gasché y R. Podestá (coords.), Educando en la Diversidad. Investigaciones y experiencias educativas interculturales y bilingües (pp. 279-366). Quito, Ecuador: Abya-Yala.

Paradise, R. (1991). El conocimiento cultural en el salón de clase: niños indígenas y su orientación hacia la observación. Infancia y Aprendizaje. México: Dialnet.

Podestá, R. (2011). Nuevas experiencias docentes de interaprendizaje en la formación de maestros indígenas. En M. Bertely (coord). Interaprendizajes entre indigenas. De cómo las y los educadores pescan conocimientos y significados comunitarios en contextos interculturales (pp. 83-116). México: CIESAS/UPN/REDIIN.

Rogoff, B. (1997). Los tres planos de la actividad sociocultural: apropiación participativa, participación guiada y aprendizaje. En J. Wertsch, P. Del Rio y A. Álvarez (eds.), La mente sociocultural: aproximaciones teóricas aplicadas (pp. 111-128). España: Fundación Infancia y aprendizaje.

Unión de Maestros de la Nueva Educación (UNEM). (2009). Modelos curriculares de educación intercultural bilingüe. México: Centro de Investigaciones y Estudio Superiores de Antropología Social (CIESAS).

Vygotsky, L. (1988). El desarrollo de los procesos psicológicos superiores. México: Editorial Crítica, Grupo editorial Grijalbo. 